\title{
Cellular Genome-scale Metabolic Modeling Identifies New Potential Drug Targets Against Hepatocellular Carcinoma
}

Oveis Jamialahmadi

University of Gothenburg

Ehsan Salehabadi

Tarbiat Modares University

Sameereh Hashemi-Najafabadi ( $\nabla$ s.hashemi@modares.ac.ir)

Tarbiat Modares University

Ehsan Motamedian

Tarbiat Modares University

Fatemeh Bagheri

Tarbiat Modares University

Rosellina Margherita Mancina

University of Gothenburg

Stefano Romeo

University of Gothenburg

\section{Research Article}

Keywords: Genome-scale metabolic model, Transcriptomic data integration, Hepatocellular carcinoma, Drug repurposing

Posted Date: December 6th, 2021

DOI: https://doi.org/10.21203/rs.3.rs-1120182/v1

License: (1) (i) This work is licensed under a Creative Commons Attribution 4.0 International License. Read Full License 


\section{Abstract}

Hepatocellular carcinoma is the third leading cause of cancer related mortality worldwide. Often this hepatic cancer is associated with fatty liver disease and insulin resistance with genetic predisposition are its major driver. Genome-scale metabolic modeling (GEM) is a promising approach to understand cancer metabolism and to identify new drug targets. Here, we used TRFBA-CORE, an algorithm generating a model using key growth-correlated reactions. Specifically, we generated a HepG2 cell-specific GEM by integrating this cell line transcriptomic data with a generic human metabolic model to predict potential drug targets for hepatocellular carcinoma (HCC).

A total of 108 essential genes for growth were predicted by TRFBA-CORE. These genes were enriched for metabolic pathways involved in cholesterol, sterols and steroids biosynthesis. Furthermore, we silenced a predicted essential gene, 11-beta dehydrogenase hydroxysteroid type 2 (HSD11B2), in HepG2 cells resulting in a reduction in cell viability. To further identify novel potential drug targets in HCC, we examined the effect of 9 drugs targeting the essential genes, and observed that most drugs inhibited the growth of HepG2 cells. Interestingly, some of these drugs in this model performed better than Sorafenib, the first line therapeutic against HCC.

\section{Introduction}

Despite the advances in the identification of somatic mutations in cancer-related genes by nextgeneration sequencing (NGS), the high heterogeneity of cancer cells, along with the complexity of biological networks, renders difficult to determine the metabolic consequences of these mutagenic events ${ }^{1,2}$.

Hepatocellular carcinoma is the third leading cause of cancer related mortality worldwide ${ }^{3}$. Often this hepatic cancer is associated with fatty liver disease and insulin resistance with genetic predisposition being its major driver ${ }^{4-6}$. Despite the presence of at least two classes of drugs as a first line therapeutic option, the survival of those affected remain poor ${ }^{7}$. Thus, there is a need to develop novel approaches to unravel the high heterogeneity of HCC tumors and identify potential drug targets 8,9 .

Genome-scale metabolic modeling is one of the growing approaches using omics data to understand how cancer metabolism responds to genetic and environmental cues ${ }^{10,11}$. General human metabolic models (GEMs) are composed of all biochemical reactions present in various human tissues and cells ${ }^{12-}$ 14 . However, due to this generality, global human GEMs are non-specific. Therefore, the integration of multiple high-throughput omics data from a specific cancerous cell type with existing general human GEMs provides a valuable tool to study cancer metabolism and to discover new drug targets and biomarkers $2,15,16$.

To date, many algorithms for context-specific reconstruction through the integration of omics data with GEMs have been developed ${ }^{17-26}$, and several studies have examined these approaches from a variety of 
perspectives, from mathematical properties to their application to peruse cancer metabolism. We recently benchmarked context-specific algorithms to study cancer metabolism, and developed TRFBA-CORE, an algorithm to reconstruct minimal cell-specific cancer metabolic models.

Here, we employed TRFBA-CORE to build a HepG2 cell-specific model by integrating transcriptomic data from this cell type and a generic human metabolic model. We next assessed the strength of the generated model in predicting cancer essential genes and further examined the knockdown effect of one of the predicted essential genes on the reduction of HepG2 cell growth. Moreover, taking a drug repurposing approach, we selected several approved drugs targeting the predicted essential genes identified by our algorithm, and examined their treatment effect on HepG2 cell viability in vitro. We specifically compared the inhibitory effect of these compounds in different concentrations to Sorafenib, a well-known approved drug for advanced HCC.

\section{Results And Discussion}

\subsection{Metabolic modeling of HCC}

Here, we employed TRFBA-CORE, a benchmark-driven algorithm developed by an extensive evaluation of other cancer genome-scale metabolic modeling methods, to find potential drug targets for $\mathrm{HCC}^{27}$. We also reconstructed two cell-specific GEMs using mCADRE and CORDA algorithms to compare their predictive capabilities with TRFBA-CORE in simulating a set of defined metabolic tasks for the liver 12,19,26. CORDA was able to predict a wider range of liver metabolic tasks (Figure 1). However, the enrichment of cell-specific models with essential genes for HepG2 showed that only GEM generated by TRFBA-CORE was able to significantly predict essential genes (Enrichment $p$ values $=0.005,0.098$, and 0.086 , for TRFBA-CORE, CORDA, and mCADRE, respectively).

The use of growth-correlated key reactions and the FASTCORE minimal modeling approach may explain the ability of TRFBA-CORE to significantly predict HepG2 essential genes. The size of generated models further shows that the TRFBA-CORE model has the minimum number of reactions required to render the network flux consistent (Figure 2).

\subsection{Identification of potential drug targets for HCC}

Of 139 essential genes predicted by TRFBA-CORE, 31 genes (22\%) disrupted defined metabolic functions in energy metabolism and redox equilibrium, or biomass production, predominantly in the OXPHOS pathway (Supplementary table S1). Therefore, we excluded these genes due to their potential adverse effects on healthy cells, and performed a gene-set enrichment analysis using the remaining 108 predicted essential genes to better understand their functional impacts. Interestingly gene ontology (GO) biological processes showed an enrichment of processes involved in cholesterol, sterols and steroids biosynthesis (Supplementary table S2). Furthermore, KEGG (Supplementary table S3) and Reactome (Supplementary table S4) biochemical pathways involved in lipid and lipoprotein metabolism, biosynthesis, cholesterol 
and steroids regulation, and carbohydrate and amino acids metabolisms had a significant overlap with the predicted essential genes.

Cholesterol is a key molecule in cell membrane structure, and a prerequisite for essential hormones and bile acids. The liver is one of the main organs for the synthesis and metabolism of endogenous cholesterol in the body ${ }^{28,29}$. The growth of HCC tumors is dependent on cholesterol biosynthesis, and the use of statins (inhibitors of HMG-CoA reductase) has been suggested as a candidate therapy for HCC. Although this enzyme is a key regulatory enzyme in cholesterol synthesis, several other enzymes in the cholesterol biosynthesis pathway have been targeted to prevent or reduce cell proliferation in various cancers ${ }^{28}$. Furthermore, previous findings suggest that the mevalonate pathway plays a role in the development of $\mathrm{HCC}^{30-32}$. Of the 18 essential genes predicted for cholesterol metabolism, 17 metabolic genes (excluding sterol 0-acyltransferase 1 (SOAT1)) has been previously identified to be involved in the production of potential antimetabolites reducing the growth of HCC tumors ${ }^{28}$.

Moreover, glucocorticoids, such as dexamethasone, are a class of steroids widely used as antiinflammatory agents. Ma et al. showed that the expression of two essential enzymes that regulate endogenous glucocorticoids and glucogenesis was altered in malignant hepatocytes, so that the expression of 11-beta dehydrogenase hydroxysteroid type 1 (HSD11B1) and 2 (HSD11B2) in mouse and human tumor cells were up and down-regulated, respectively. Moreover, the expression ratio of HSD11B1:HSD11B2 was related to the prognosis and survival of patients with HCC, so that patients with a high ratio of HSD11B1: HSD11B2 had a longer survival time than others ${ }^{33,34}$. Also, it has been shown that HSD11B1 expression usually leads to a decrease in cell proliferation, while HSD11B2 expression is involved in an increase in cell proliferation. Several studies showed that the expression of these two genes varies in different tumors and can provide a suitable microenvironment for tumor growth ${ }^{35,36}$. Therefore, HSD11B2, which was the only predicted essential gene in the steroid biosynthesis pathway, was selected for further investigation.

We compared the relative expression level of HSD11B2 in HepG2 and three non-cancerous cell lines, namely HH, HHSEC and LX-2 (Figure 3a) and observed a relatively higher expression of HSD11B2 in HepG2 cells. Therefore, we silenced HSD11B2 in HepG2 cells (Figure 3b) to examine its knockdown effect on the cell viability. Interestingly, we were able to observe a modest but significant reduction in cell viability after $48(p$-value $=0.021)$ and $72(p$-value $=6.5 \mathrm{E}-4)$ hr (Figure 4$)$

Glucocorticoids are essential hormones in the body, regulating the nuclear expression of approximately $5 \%$ of human genes. One of their function is to increase glucose synthesis by increasing gluconeogenesis. Ma et al. showed that glycogenesis is impaired in HCC, and that the abnormal expression of two genes, HSD11B1 and HSD11B2, affects some key genes in the glucose pathway. Although the mechanism by which malignant hepatocytes target these two genes for malignant progression is not clear, targeting HSD11B2 in a mouse model reduced tumor mass ${ }^{34}$. Furthermore, by comparing the RNA-Seq expression data in GEPIA web server, we observed that HSD11B1 and HSD11B2 were respectively down- and up-regulated in HCC samples $(P<0.05)$. The expression of these two genes 
showed a negative correlation in healthy and cancerous samples from Atlas of Cancer Genome (TCGA, Spearman $R=-0.18, p=3 \times 10^{-4}$ ), while, the correlation in healthy samples was positive (Spearman $R=0.36$, $p=0.011$ ). Therefore, given the lower expression of HSD11B1 in HCC cells, HSD11B2 may be a potential target gene in HCC to regulate the HSD11B1: HSD11B2 expression ratio ${ }^{37,38}$.

\subsection{Drug repositioning for HCC}

Drug repurposing is an attractive approach providing cancer patients with faster and cost-effective potential therapeutics with well-characterized safety profiles ${ }^{39,40}$. Here, we used the DrugBank database to evaluate potential compounds targeting the predicted metabolic essential genes ${ }^{41}$. Thus, a set of metabolic drugs targeting one or more of the predicted genes was obtained, among which we selected 9 compounds by reviewing the literature and applying the following criteria; first, metabolic drug has not been used in the treatment of HCC, and secondly, has been evaluated in at least one of the other cancers, or the target gene was suggested as a therapeutic target for HCC (Table 1).

Table 1 Drugs reviewed in this study. Only intersection of predicted essential genes and targets of each therapeutic compound has been shown in metabolic targets column.

\begin{tabular}{|c|c|c|c|}
\hline Subsystems & $\begin{array}{l}\text { Metabolic } \\
\text { targets }\end{array}$ & $\begin{array}{l}\text { DrugBank accession } \\
\text { number }\end{array}$ & Drug \\
\hline Cholesterol Metabolism & CYP51A1 & DB01007 & Tioconazole \\
\hline Nucleotides & RRM1 & DB00631 & Clofarabine \\
\hline Nucleotides & TYMS & DB00432 & Trifluridine \\
\hline Pyrimidine Biosynthesis & DHODH & DB01117 & Atovaquone \\
\hline Nucleotides & ADSSL1; ADSS & DB05540 & Alanosine \\
\hline $\begin{array}{l}\text { Folate Metabolism; } \\
\text { Nucleotides }\end{array}$ & DHFR; TYMS & DB06813 & Pralatrexate \\
\hline Cholesterol Metabolism & FDPS & DB00710 & Ibandronate \\
\hline Cholesterol Metabolism & SQLE & DB00857 & Terbinafine \\
\hline Cholesterol Metabolism & FDPS & DB00630 & $\begin{array}{l}\text { Alendronic } \\
\text { Acid }\end{array}$ \\
\hline \multicolumn{4}{|c|}{$\begin{array}{l}\text { We next examined the treatment effect of these compounds on HepG2 cell viability in } 5 \text { different } \\
\text { concentrations. Interestingly, most of the drugs, except Terbinafine, were able to significantly reduce the } \\
\text { cell viability (Figure 5). In addition, some of these drugs showed a comparatively higher inhibitory effect } \\
\text { compared to Sorafenib, the well-known approved drug to treat advanced HCC. For instance, Clofarabine, } \\
\text { Alanosine, and Pralatrexate were more effective than Sorafenib at lower concentrations ( } 100 \text { and } 1 \mathrm{~nm} \text { ). } \\
\text { Alanosine targeted two genes adenylosuccinate synthetase like } 1 \text { (ADSSL1) and adenylosuccinate } \\
\text { synthetase (ADSS), which TRFBA-CORE predicted as synthetic lethal. These two genes encode two }\end{array}$} \\
\hline
\end{tabular}


isozymes, adenylosuccinate synthase 1 and 2, respectively, catalyzing a key reaction in purine nucleotide biosynthesis. The effect of Alanosine on inhibiting the growth of other cancerous cells has also been demonstrated ${ }^{42}$. Targeting AMP, another critical precursor by Alanosine, has also been shown to be effective at the nucleotide levels ${ }^{43}$.

AMP is produced from the IMP by ADSS and ADSSL1, and an essential precursor in the production of DNA and RNA ${ }^{44}$. Synthetic lethality occurs in a cell when perturbations of two genes combined are lethal, while a genetic defect in either gene alone is harmless. In this case, a genetic change, such as a defect in one tumor suppressor gene causes another gene to become essential for cell viability, and therefore, offering a therapeutic approach by selective targeting of cancer cells ${ }^{45}$. TRFBA-CORE predicted a total of 25 synthetic lethal pairs, nine of which had previously been identified in the study of Folger et al. ${ }^{46}$. Interestingly, none of these predicted gene pairs disrupted the defined metabolic functions in Recon 1.

Among the novel predicted synthetic lethal pairs, hydroxyl methylglutaryl-CoA synthase (HMGCS2), which is involved in cholesterol metabolism, contained eight gene pairs all of which were involved in amino acid metabolism (Supplementary table S5). Moreover, carnitine 0-acyl transferase (CRAT), which facilitates the release of acetyl-CoA mitochondria into the cytosol ${ }^{47}$, had three gene pairs that were involved in TCA and citrate transport from mitochondria to the cytosol. The role of HMGCS2 and CRAT in several cancers has been already shown ${ }^{47,48}$.

In addition to Alanosine, Clofarabine, one of the most successful drugs against leukemia ${ }^{49}$, targets the large subunit of ribonucleoside-diphosphate reductase (RRM1). Notably, few other drugs targeting RRM1 have previously been suggested to inhibit HCC cell growth and other liver diseases ${ }^{50,51}$. RRM1 and RRM2 are responsible for ribonucleotide reductase expression, which acts as a rate-limiting enzyme in dNTP production. Also, Pralatrexate targets dihydrofolate reductase (DHFR) and thymidylate synthetase (TYMS), both of which were predicted to be essential genes. Pralatrexate is an approved drug in the treatment of peripheral T cell lymphoma, and its promising outcome has been shown in some other cancer cells ${ }^{52,53}$. However, the effect of Pralatrexate on growth was relatively constant at different concentrations (Figure 5). TYMS plays a vital role in dTMP synthesis, which is significantly upregulated in tumor cells ${ }^{54}$. Tetrahydrofolate (THF), the biologically active form of folic acid, is an essential metabolite synthetized by DHFR and utilized by TYMS to produce dTMP, a critical compound in DNA replication ${ }^{55,56}$.

Interestingly, Trifluridine had a larger inhibitory effect on cell growth than Sorafenib at a low concentration of $1 \mu \mathrm{m}$. Trifluridine/ tipiracil combination therapy has been shown to have a beneficial effect on survival in those with colon cancer ${ }^{57}$. Furthermore, several other drugs targeting TYMS have been suggested for the treatment of HCC 58,59 .

Notably, while Sorafenib showed a relatively stronger inhibitory effect on cell viability at $10 \mu \mathrm{m}$, thioconazole, an imidazole antifungal drug, showed a larger effect compared to the other compounds at 
higher concentrations $(25 \mu \mathrm{m}$ and $50 \mu \mathrm{m}$, Figure 5$)$. One of the drug targets of thioconazole is lenosterol 14-alpha-demethylase (CYP51A1), which has been linked to the size of HCC tumors in the carriers of the hepatitis $C$ virus ${ }^{60}$. Recently, the effect of thioconazole on inhibiting the growth of cancer cells has been shown ${ }^{61}$. Moreover, at these concentrations, the inhibitory effect of Alendronic acid, Alanosine, Ibandronate and Atovaquone, on growth was similar to that of Sorafenib. Alendronic acid and Ibandronate both inhibit farnesyl pyrophosphate synthase (FDPS). Pamidronic acid and zoldronic acid, both of which are FDPS inhibitors, have also shown antiproliferative effects in $\mathrm{HCC}{ }^{62,63}$. Moreover, the efficacy of Atovaquone, an antimalarial drug, has recently been demonstrated in various cancers ${ }^{64,65}$. Atovaquone inhibits the activity of dihydroorotate dehydrogenase (DHODH), which is involved in de novo pyrimidine synthesis, resulting in a forced pause of DNA and RNA synthesis in cancer cells ${ }^{66-68}$.

\section{Materials And Methods}

\subsection{General human model set-up}

The consistent Recon 1 general model (i.e. after removal of all blocked reactions incapable of carrying a non-zero flux) was used to generate context-specific models ${ }^{13,23,46}$. It has been shown that alanine and glutamate are secreted (but not uptaken) by cancer cells; therefore, the corresponding exchange reactions were not constrained in the input model ${ }^{69,70}$. TRFBA-CORE algorithm was used to generate a functional cell-specific model from HepG2 expression data for HepG2 cells (taken from the Gene Expression Omnibus (GEO) public repository under the accession number GSE38124) ${ }^{71,72}$. The generated cellspecific GEM was then constrained with simulated MEM $+10 \%$ FBS medium ${ }^{46,73-75}$ to be consistent with in-vitro experiments.

\subsection{Liver metabolic functions}

A set of 256 previously defined metabolic functions (such as the synthesis of fatty acids, amino acids, cholesterol, and bile acids), which are physiologically relevant to healthy hepatocytes, were used to validate the generated cell-specific GEM ${ }^{9,12}$. We kept only a set of 197 tasks that could be performed by consistent Recon 1, and GEM performance was reported as the fraction of successfully passed metabolic functions. To further evaluate the performance of the generated cell-specific model, two additional cellspecific GEMs were generated using CORDA and mCADRE algorithms. CORDA ${ }^{26}$ uses a dependency assessment to minimize the flux of cost-consuming reactions, and only the non-key reactions required for the final network activity are considered. Similarly, mCADRE reconstructs context-specific modes by using gene expression and network topology to score all reactions in a model ${ }^{19,27}$.

\subsection{Prediction of essential genes}

Gene dependence scores calculated by the DEMETER2 algorithm for the HepG2 cell line were obtained from the DepMap database ${ }^{76}$. The DEMETER2 algorithm analyzes the RNA interference (RNAi) screens, reduces the off-target effects of RNAi and corrects for confounding factors such as batch effects ${ }^{76,77}$. 
Here, the genes with a DEMETER2 dependence score below zero were considered as being essential. Finally, a hypergeometric enrichment test was used to evaluate the over-representation of essential genes present in each of the generated GEMs.

To account for possible side-effects of the predicted essential genes, we used a set of 56 metabolic tasks occurring in all cell types ${ }^{28}$,therefore, we excluded genes that disrupted any of the tasks involved in energy metabolism and redox balance, or biomass production ${ }^{78}$. Double gene deletion simulation was also performed to identify synthetic lethal genes. A synergy score was defined as $\mathrm{KO}_{\mathrm{AB}} / \min \left(\mathrm{KO}_{\mathrm{A}}, \mathrm{KO}_{\mathrm{B}}\right)$; where $\mathrm{KO}_{\mathrm{A}}, \mathrm{KO}_{\mathrm{B}}$, and $\mathrm{KO}_{\mathrm{AB}}$ are simulated growth after the repression of gene $\mathrm{A}$, gene $\mathrm{B}$, and simultaneous suppression of genes $A$ and $B$, respectively ${ }^{46}$. Finally, double genes with a synergy score greater than 0.5 were selected as synthetic lethal ${ }^{46}$. Similar to single gene deletion simulations, double gene deletions that perturbed any of the above-mentioned metabolic functions were excluded.

Predicted essential genes were further used to perform gene ontology (GO) biological processes, Kyoto Encyclopedia of Genes and Genomes (KEGG) and Reactome biochemical pathways enrichment analysis using Enrichr. Adjusted $\mathrm{P}$ values and combined scores (log $\mathrm{P}$ value $\times \mathrm{z}$-score) were reported ${ }^{79}$.

\subsection{Cell culture}

HepG2 (hepatocellular carcinoma) derived from the liver tissue of a 15-year-old Caucasian male who had a well-differentiated hepatocellular carcinoma were purchased from ATCC (ATCC $\AA$ HB8065 ${ }^{\mathrm{TM}}$ ). After thawing, the cells were plated in T-75 flasks and cultured at $37^{\circ} \mathrm{C}$ in Minimum Essential Medium (MEM), containing Fetal Bovine Serum (FBS) 10\%, L-glutamine $2 \mathrm{mM}$, sodium pyruvate $1 \mathrm{mM}$ and non-essential amino acids $1 \mathrm{X}$ (NEAA) in a humidified atmosphere of $5 \% \mathrm{CO} 2$.

\subsection{Gene expression}

Total RNA of HepG2 cells was extracted by the RNeasy Mini kit (Qiagen, Valencia, CA) and first-strand cDNA was synthesized using the High Capacity cDNA Reverse Transcription Kit (Thermo Fisher Scientific) according to the manufacturer's instructions. HSD11B2 expression in HepG2, human hepatocytes $(\mathrm{HH})$, Human Hepatic Sinusoidal Endothelial Cells (HHSEC), and Human Hepatic Stellate Cells (LX-2) (Sciencell, Carlsbad, CA) was measured using TaqMan probes and Master Mix (Thermo Fisher Scientific) using quantitative real-time PCR (qPCR) according to the manufacturer's protocol. Expression levels were determined using the $2^{-\triangle \triangle C T}$ method and normalized to $\beta$-actin in triplicates ${ }^{80,81}$.

\subsection{Gene silencing}

HepG2 were transfected with small interfering RNA (siRNA) or negative control (scrambled) siRNA (Ambion-Life Technologies by Thermo Fisher Scientific, Rockford, IL) with Lipofectamine Transfection Reagent (Life Technologies, Carlsbad, CA) according to the manufacturer's instructions. The cells were first cultured in 6-well plates $(3 \times 105$ cells/well) for one day, and transfected with $50 \mathrm{nM}$ siRNA oligos in a serum-free medium at 50-70\% confluency. After 48 h, the RNA was extracted by the RNeasy Mini Kit 
(Qiagen, Hilden, Germany), and after cDNA synthesis, t HSD11B2 knockdown efficiency was confirmed. All experiments were performed in triplicates.

\subsection{Cell viability assay}

To observe the effect of gene knockdown on cell growth, cell viability was investigated by CellTiter $96 \AA$ AQueous One Solution Assay (Promega, Madison, WI, USA). This assay is based on the ability of living cells to reduce the tetrazolium, 3- (4, 5-dimethylthiazol-2-yl) -5- (3-carboxymethoxyphenyl) -2- (4sulfophenyl) $-2 \mathrm{H}$-tetrazolium (MTS), to formazan. For this purpose, at 24,48 , and 72 hours after knockdown, the culture medium was changed, and $20 \%$ of the volume of the culture medium containing cells, MTS solution was added to the wells containing transfected cells. After incubation for 1 hour, the absorbance of the samples was measured at $490 \mathrm{~nm}$ in a microplate reader. All experiments were performed at least three times, with five repetitions each time.

To evaluate the treatment effect of candidate drugs on cell viability, HepG2 cells were cultured at a density of 5000 cells/well in a 96-well plate, and after incubating for overnight, the cells were treated with each drug at 5 different concentrations ( $100 \mathrm{~nm}, 1 \mu \mathrm{m}, 10 \mu \mathrm{m}, 25 \mu \mathrm{m}$ and $50 \mu \mathrm{m}$ in $0.5 \%$ DMSO). Cells treated with $0.5 \%$ DMSO were used as control. All experiments were performed in triplicates. After 72 and 96 hours, cell viability was measured as described above.

\section{Limitations}

This study has at least two limitations: a) the use of a single cell type, namely HepG2, and results may be driven by the specific genetic background of these cells deriving from a single donor. For example, they are homozygotes for the PNPLA3 I148M genetic variant, the strongest common inborn genetic risk factor predisposing to hepatocellular carcinoma $3,82,83 ; b$ ) the efficacy of the compounds should be confirmed in other cell types and in murine models of HCC.

\section{Conclusion}

Here, we used our recently developed algorithm, TRFBA-CORE, and by integrating HepG2 expression data and a general human model, generated a specific cell model for HepG2. Many of the reactions present in this minimal HepG2 model overlapped with model content of the two models generated by mCADRE and CORDA. However, the use of growth correlation reactions by TRFBA-CORE improved the capability of the model in predicting essential HepG2 genes.

We further confirmed that the knocking down of one predicted essential gene, HSD11B2, significantly reduced HepG2 cell growth. Previous findings suggest that the expression of this gene is directly related to the growth of mouse liver cancer cells. Hence, upon confirmation on other cell types, HSD11B2 can be a potential target for further studies in HCC monitoring and inhibition. 
We also examined the in vitro effect of several drugs targeting the predicted essential genes on HepG2 cell growth, and observed that most of the selected drugs show a significant inhibitory effect on cell growth in a wide range of concentrations. Notably, several drugs were more effective than Sorafenib, the first line treatment against HCC treatment.

Overall, our findings highlight the potential of metabolic modeling to offer novel therapeutics against liver cancer cell growth. Nevertheless, future molecular and pharmacological studies are warranted to elucidate the role these compounds and their potential against HCC.

\section{Declarations}

Data availability All data generated or analyzed during this study are available.

Code availability Not applicable

Authors' contributions All authors included in this paper fulfill the criteria of authorship and have approved the submission of this manuscript. S. Hashemi-Najafabadi, E. Motamedian and S. Romeo supervised the study. O. Jamialahmadi, F. Bagheri, and R. M. Mancina designed the experiments and data analysis. O. Jamialahmadi, E. Salehabadi, S. Hashemi-Najafabadi, E. Motamedian and S. Romeo wrote the manuscript. O. Jamialahmadi and E. Salehabadi contributed equally to this work.

Ethics approval and consent to participate Not applicable

Funding Stefano Romeo was supported by project grants from the Swedish Research Council (Vetenskapsrådet [VR], 2016-01527), the Swedish state under the agreement between the Swedish government and the county councils (the ALF agreement) (SU 2018-04276), the Swedish Diabetes Foundation (DIA 2017-205), the Swedish Heart-Lung Foundation (20120533), the Wallenberg Academy Fellows from the Knut and Alice Wallenberg Foundation (KAW 2017.0203), the Astra Zeneca Agreement for Research, Grant SSF ITM17-0384 Swedish Foundation for Strategic Research, and Novo Nordisk Project Grants in Endocrinology \& Metabolism - Nordic Region 2020.

Consent for publication All authors have agreed to publish this manuscript.

Competing interests The authors declare no competing interests. Stefano Romeo has served as a consultant for AstraZeneca, Celgene, Sanofi, Amgen, Akcea Therapeutics, Camp4, AMbys, Medacorp and Pfizer in the past 5 years, and received research grants from AstraZeneca, Sanofi and Amgen.

\section{References}

1. Lewis, N. E. \& Abdel-haleem, A. M. The evolution of genome-scale models of cancer metabolism. Front Physiol, 4, 1-7 (2013). 
2. Yizhak, K., Chaneton, B., Gottlieb, E. \& Ruppin, E. Modeling cancer metabolism on a genome scale. Mol Syst Biol, 11, 1-17 (2015).

3. Bianco, C. et al. Non-invasive stratification of hepatocellular carcinoma risk in non-alcoholic fatty liver using polygenic risk scores. J. Hepatol, 74, 775-782 (2021).

4. Romeo, S., Sanyal, A. \& Valenti, L. Leveraging Human Genetics to Identify Potential New Treatments for Fatty Liver Disease. Cell Metab, 31, 35-45 (2020).

5. Loomba, R., Friedman, S. L. \& Shulman, G. I. Mechanisms and disease consequences of nonalcoholic fatty liver disease., 184, 2537-2564 (2021).

6. Valenti, L., Pedica, F. \& Colombo, M. Distinctive features of hepatocellular carcinoma in non-alcoholic fatty liver disease. Dig. Liver Dis, https://doi.org/doi:10.1016/j.dld.2021.06.023. (2021).

7. Vitale, A., Trevisani, F., Farinati, F. \& Cillo, U. Treatment of Hepatocellular Carcinoma in the Precision Medicine Era: From Treatment Stage Migration to Therapeutic Hierarchy., 72, 2206-2218 (2020).

8. Bidkhori, G. et al. Metabolic Network-Based Identification and Prioritization of Anticancer Targets Based on Expression Data in Hepatocellular Carcinoma. Front. Physiol, 9, 916 (2018).

9. Mukhopadhyay, B. et al. Stratification of Hepatocellular Carcinoma Patients Based on Acetate Utilization. Cell Rep, 13, 2014-2026 (2015).

10. Nilsson, A. \& Nielsen, J. Genome scale metabolic modeling of cancer. Metab. Eng, 43, 103-112 (2017).

11. Hyduke, D. R., Lewis, N. E. \& Palsson, B. Ø. Analysis of omics data with genome-scale models of metabolism. Mol. BioSyst. 9, 167-174 (2013)

12. Mardinoglu, A. et al. Genome-scale metabolic modelling of hepatocytes reveals serine deficiency in patients with non-alcoholic fatty liver disease. Nat. Commun, 5, 1-11 (2014).

13. Duarte, N. C. et al. Global reconstruction of the human metabolic network based on genomic and bibliomic data. Proc. Natl. Acad. Sci. U. S. A. 104, 1777-1782(2007)

14. Swainston, N. et al. Recon 2.2: from reconstruction to model of human metabolism.Metabolomics12, (2016)

15. Jerby, L. \& Ruppin, E. Predicting Drug Targets and Biomarkers of Cancer via Genome-Scale Metabolic Modeling. Clin Cancer Res, 18, 5572-5585 (2012).

16. Ryu, J. Y., Kim, H. U. \& Lee, S. Y. Reconstruction of genome-scale human metabolic models using omics data. Integr. Biol, 7, 859-868 (2015).

17. Yizhak, K. et al. Phenotype-based cell-specific metabolic modeling reveals metabolic liabilities of cancer. Elife, 3, 1-23 (2014).

18. Pacheco, M. P. et al. Integrated metabolic modelling reveals cell-type specific epigenetic control points of the macrophage metabolic network. BMC Genomics, 16, 1-24 (2015).

19. Wang, Y., Eddy, J. A. \& Price, N. D. Reconstruction of genome-scale metabolic models for 126 human tissues using mCADRE. BMC Syst Biol, 6, 153 (2012). 
20. Jerby, L., Shlomi, T. \& Ruppin, E. Computational reconstruction of tissue-specific metabolic models: application to human liver metabolism. Mol. Syst. Biol, 6, 1-9 (2010).

21. Agren, R. et al. Reconstruction of Genome-Scale Active Metabolic Networks for 69 Human Cell Types and 16 Cancer Types Using INIT.PLoS Comput Biol.8, (2012)

22. Shlomi, T., Cabili, M. N., Herrgård, M. J., Palsson, B. Ã. \& Ruppin, E. Network-based prediction of human tissue-specific metabolism. Nat. Biotechnol, 26, 1003-1010 (2008).

23. Vlassis, N., Pacheco, M. P. \& Sauter, T. Fast Reconstruction of Compact Context-Specific Metabolic Network Models. PLoS Comput Biol.10, (2014)

24. Becker, S. A. \& Palsson, B. O. Context-specific metabolic networks are consistent with experiments. PLoS Comput. Biol.4, (2008)

25. Motamedian, E., Mohammadi, M., Shojaosadati, S. A. \& Heydari, M. T. R. F. B. A. An algorithm to integrate genome-scale metabolic and transcriptional regulatory networks with incorporation of expression data., 33, 1057-1063 (2017).

26. Schultz, A. \& Qutub, A. A. Reconstruction of Tissue-Specific Metabolic Networks Using CORDA. PLoS Comput Biol, 12, 1-33 (2016).

27. Jamialahmadi, O., Hashemi-Najafabadi, S., Motamedian, E., Romeo, S. \& Bagheri, F. A benchmarkdriven approach to reconstruct metabolic networks for studying cancer metabolism. PLoS Comput. Biol.15, (2019)

28. Agren, R. et al. Identification of anticancer drugs for hepatocellular carcinoma through personalized genome-scale metabolic modeling. Mol Syst Biol, 10, 1-13 (2014).

29. Ioannou, G. N. The Role of Cholesterol in the Pathogenesis of NASH. Trends Endocrinol. Metab, 27, 84-95 (2016).

30. De Matteis, S. et al. Aberrant Metabolism in Hepatocellular Carcinoma Provides Diagnostic and Therapeutic Opportunities. Oxid. Med. Cell. Longev. 2018, 7512159 (2018)

31. Liang, J. Q. et al. Dietary cholesterol promotes steatohepatitis related hepatocellular carcinoma through dysregulated metabolism and calcium signaling. Nat Commun, 9, 4490 (2018).

32. Mullen, P. J., Yu, R., Longo, J., Archer, M. C. \& Penn, L. Z. The interplay between cell signalling and the mevalonate pathway in cancer. Nat. Rev. Cancer, 16, 718-731 (2016).

33. Ray, K. Hepatocellular carcinoma: Restoring gluconeogenesis: steroids could treat liver cancer. Nat. Publ. Gr, 10, 693 (2013).

34. Ma, R. et al. Switch of glycolysis to gluconeogenesis by dexamethasone for treatment of hepatocarcinoma. Nat. Commun, 4, 2508 (2013).

35. Azher, S. et al. The Non-Conventional Effects of Glucocorticoids in Cancer. J. Cell. Physiol, 231, 2368-2373 (2016).

36. Lu, L. et al. Expression of 11ß-hydroxysteroid Dehydrogenase Type 1 in Breast Cancer and Adjacent Non-Malignant Tissue. An Immunocytochemical Study. Pathol. Oncol. Res, 17, 627-632 (2011). 
37. Tang, Z. et al. GEPIA: a web server for cancer and normal gene expression profiling and interactive analyses. Nucleic Acids Res, 45, 98-102 (2017).

38. Li, T. et al. TIMER: A Web Server for Comprehensive Analysis of Tumor-Infiltrating Immune Cells. Cancer Res, 77, 108-111 (2017).

39. Bertolini, F., Sukhatme, V. P. \& Bouche, G. Drug repurposing in oncology-patient and health systems opportunities. Nat. Rev. Clin. Oncol, 12, 732-742 (2015).

40. Gupta, S. C., Sung, B., Prasad, S., Webb, L. J. \& Aggarwal, B. B. Cancer drug discovery by repurposing: teaching new tricks to old dogs. Trends Pharmacol. Sci, 34, 508-517 (2013).

41. Wishart, D. S. et al. DrugBank 5.0: a major update to the DrugBank database for 2018. Nucleic Acids Res, 46, 1074-1082 (2018).

42. Efferth, T., Gebhart, E., Ross, D. D. \& Sauerbrey, A. Identification of gene expression profiles predicting tumor cell response to l-alanosine. Biochem. Pharmacol, 66, 613-621 (2003).

43. Gooding, J. R. et al. Adenylosuccinate Is an Insulin Secretagogue Derived from Glucose-Induced Purine Metabolism Article Adenylosuccinate Is an Insulin Secretagogue Derived from GlucoseInduced Purine Metabolism. CellReports, 13, 157-167 (2015).

44. Barfeld, S. J. et al. Myc-dependent purine biosynthesis affects nucleolar stress and therapy response in prostate cancer. Oncotarget, 6, 12587-602 (2015).

45. Ashworth, A. \& Lord, C. J. Synthetic lethal therapies for cancer: what's next after PARP inhibitors? Nat. Rev. Clin. Oncol, 15, 564-576 (2018).

46. Folger, O. et al. Predicting selective drug targets in cancer through metabolic networks. Mol. Syst. Biol, 7, 501 (2011).

47. Anna, M. et al. The carnitine system and cancer metabolic plasticity. Cell Death Dis, 9, 228 (2018).

48. Chen, S. et al. HMGCS2 enhances invasion and metastasis via direct interaction with PPARa to activate Src signaling in colorectal cancer and oral cancer. Oncotarget, 8, 22460-22476 (2017).

49. Bonate, P. L. et al. Discovery and development of clofarabine: a nucleoside analogue for treating cancer. Nat. Rev. Drug Discov, 5, 855-863 (2006).

50. Chua, M., Bernstein, L. R., Li, R. \& So, S. K. S. Gallium Maltolate is a Promising Chemotherapeutic Agent for the Treatment of Hepatocellular Carcinoma. Anticancer Res, 1744, 1739-1743 (2006).

51. Nozaki, A. et al. Hydroxyurea as an inhibitor of hepatitis C virus RNA replication. Arch. Virol, 155, 601-605 (2010).

52. Serova, M. et al. Single agent and combination studies of pralatrexate and molecular correlates of sensitivity. Br J Cancer, 104, 272-280 (2011).

53. Marchi, E., Mangone, M., Zullo, K. \& Connor, O. A. O. Pralatrexate Pharmacology and Clinical Development. Clin Cancer Res, 19, 6657-6662 (2013).

54. Furuta, E., Okuda, H., Kobayashi, A. \& Watabe, K. Metabolic genes in cancer: Their roles in tumor progression and clinical implications. Biochim. Biophys. Acta - Rev. Cancer, 1805, 141-152 (2010). 
55. He, Y. et al. Metabolic Intermediates in Tumorigenesis and Progression. Int J Biol Sci, 15, 1187-1199 (2019).

56. Raimondi, M. V. et al. DHFR Inhibitors: Reading the Past for Discovering Novel Anticancer Agents. Molecules, 24, 1-19 (2019).

57. Mayer, R. J. et al. Randomized Trial of TAS-102 for Refractory Metastatic Colorectal Cancer. N Engl J Med, 372, 1909-1919 (2015).

58. Pelizzaro, F. et al. Capecitabine in advanced hepatocellular carcinoma: A multicenter experience. Dig. Liver Dis, 51, 1713-1719 (2019).

59. Zhao, H., Zhang, Y., Sun, J., Zhan, C. \& Zhao, L. Raltitrexed Inhibits HepG2 Cell Proliferation via G0/G1 Cell Cycle Arrest. Oncol Res, 23, 237-248 (2021).

60. Tsunedomi, R. et al. Patterns of expression of cytochrome P450 genes in progression of hepatitis C virus-associated hepatocellular carcinoma. Int J Oncol, 27, 661-667 (2005).

61. Liu, P. et al. Drug Repurposing Screening Identifies Tioconazole as an ATG4 Inhibitor that Suppresses Autophagy and Sensitizes Cancer Cells to Chemotherapy. Theranostics, 8, 830-845 (2018).

62. Wada, A. et al. Pamidronate induced anti-proliferative, apoptotic, and anti-migratory effects in hepatocellular carcinoma. J Hepatol, 44, 142-150 (2006).

63. Katamura, Y. et al. Zoledronic acid delays disease progression of bone metastases from hepatocellular carcinoma. Hepatol. Res, 40, 1195-1203 (2010).

64. Fiorillo, M. et al. Repurposing atovaquone: Targeting mitochondrial complex III and OXPHOS to eradicate cancer stem cells. Oncotarget, 7, 34084-99 (2016).

65. Xiang, M. et al. Gene expression - based discovery of atovaquone as a STAT3 inhibitor and anticancer agent. Blood, 128, 1845-1853 (2016).

66. Li, L. et al. Identification of DHODH as a therapeutic target in small cell lung cancer.Sci Trans/ Med.11, (2019)

67. Sykes, D. B. The emergence of dihydroorotate dehydrogenase (DHODH) as a therapeutic target in acute myeloid leukemia in acute myeloid leukemia. Expert Opin. Ther. Targets, 22, 893-898 (2018).

68. Ashton, T. M. et al. The anti-malarial atovaquone increases radiosensitivity by alleviating tumour hypoxia.Nat. Commun.7, (2016)

69. Khazaei, T., Mcguigan, A. \& Mahadevan, R. Ensemble modeling of cancer metabolism. Front Physiol, 3, 1-14 (2012).

70. Jain, M. et al. Metabolite Profiling Identifies a Key Role for Glycine in Rapid Cancer Cell Proliferation. Science (80-), 336, 1040-1044 (2012).

71. Rieswijk, L., Lizarraga, D., Brauers, K. J. J., Kleinjans, J. C. S. \& Delft, J. H. M. Van. Characterisation of cisplatin-induced transcriptomics responses in primary mouse hepatocytes, HepG2 cells and mouse embryonic stem cells shows conservation of regulating transcription factor networks. Mutagenesis, 29, 17-26 (2013). 
72. Edgar, R., Domrachev, M. \& Lash, A. E. Gene Expression Omnibus: NCBI gene expression and hybridization array data repository. Nucleic Acids Res, 30, 207-210 (2002).

73. Auslander, N. et al. An integrated computational and experimental study uncovers FUT 9 as a metabolic driver of colorectal cancer. Mol. Syst. Biol, 13, 956 (2017).

74. Agren, R. et al. The RAVEN Toolbox and Its Use for Generating a Genome-scale Metabolic Model for Penicillium chrysogenum. PLoS Comput. Biol.9, (2013)

75. Fouladiha, H., Marashi, S. A. \& Shokrgozar, M. A. Reconstruction and validation of a constraint-based metabolic network model for bone marrow-derived mesenchymal stem cells. Cell Prolif, 48, 475-485 (2015).

76. Mcfarland, J. M. et al. Improved estimation of cancer dependencies from large-scale RNAi screens using model-based normalization and data integration. Nat. Commun, 9, 1-13 (2018).

77. Tseng, Y. Y. \& Boehm, J. S. From cell lines to living biosensors: new opportunities to prioritize cancer dependencies using ex vivo tumor cultures. Curr. Opin. Genet. Dev, 54, 33-40 (2019).

78. Ghaffari, P. et al. Identifying anti-growth factors for human cancer cell lines through genome-scale metabolic modeling. Sci Rep, 5, 1-10 (2015).

79. Kuleshov, M. V. et al. Enrichr: a comprehensive gene set enrichment analysis web server 2016 update. Nucleic Acids Res, 44, 90-97 (2016).

80. Holland, P. M., Abramson, R. D., Watson, R. \& Gelfand, D. H. Detection of specific polymerase chain reaction product by utilizing the 5'---3' exonuclease activity of Thermus aquaticus DNA polymerase. Proc. Natl. Acad. Sci. 88, 7276-7280 (1991)

81. Livak, K. J. \& Schmittgen, T. D. Analysis of Relative Gene Expression Data Using Real-Time Quantitative PCR and the $2-\Delta \Delta$ CT Method., 25, 402-408 (2001).

82. Burza, M. A. et al. <em>PNPLA3</em> I148M (rs738409) genetic variant is associated with hepatocellular carcinoma in obese individuals. Dig. Liver Dis, 44, 1037-1041 (2012).

83. Pingitore, P. \& Romeo, S. The role of PNPLA3 in health and disease. Biochim. Biophys. Acta - Mol. Cell Biol. Lipids, 1864, 900-906 (2019).

\section{Figures}




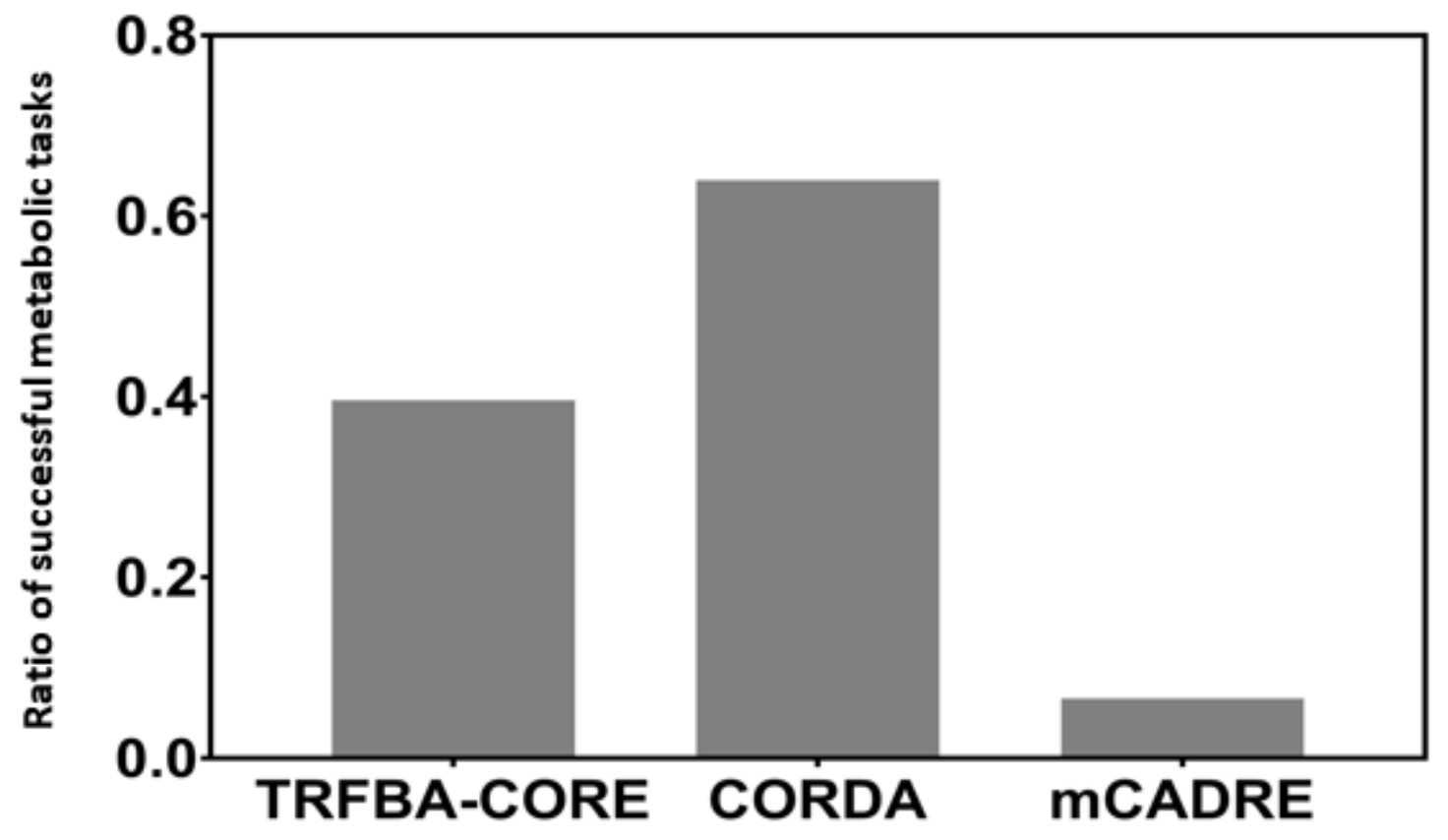

Figure 1

Simulation of liver metabolic functions for HepG2 cell-specific models. The results are reported as the ratio of successful tasks by each model to those of the consistent Recon 1 (a total of 197 metabolic tasks).

a

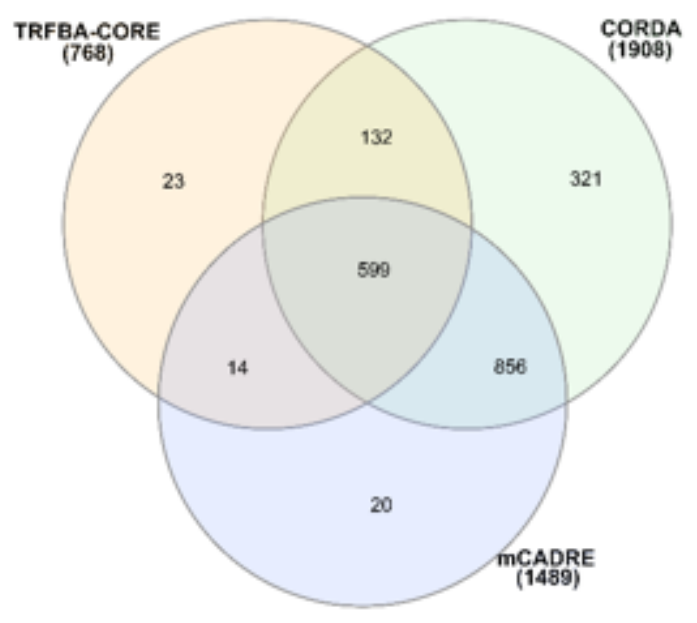

b

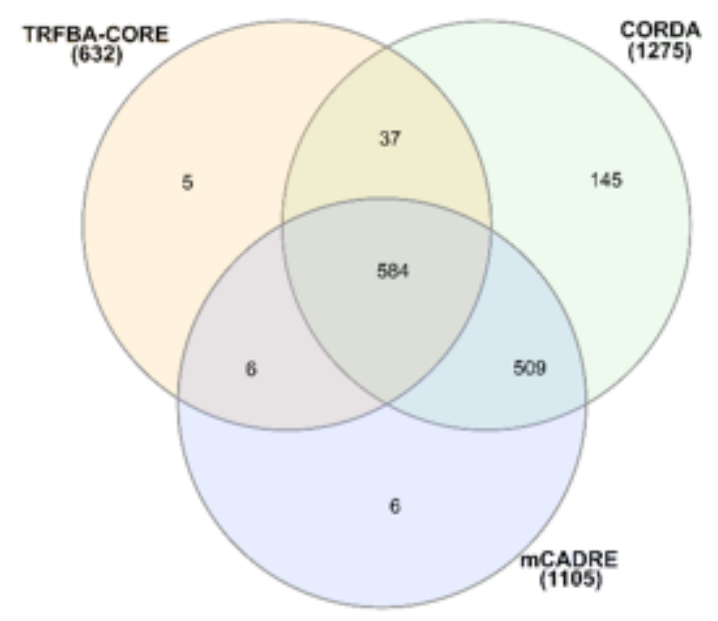

Figure 2

Comparison of the size of HepG2 cell-specific models. The Venn diagrams show the overlap between (a) reactions and (b) metabolites present in the three cell-specific models generated by TRFBA-CORE, CORDA, 
and mCADRE. Most of the reactions (95\%) and metabolites (98\%) of the TRFBA-CORE cell-specific model were present in CORDA.

a

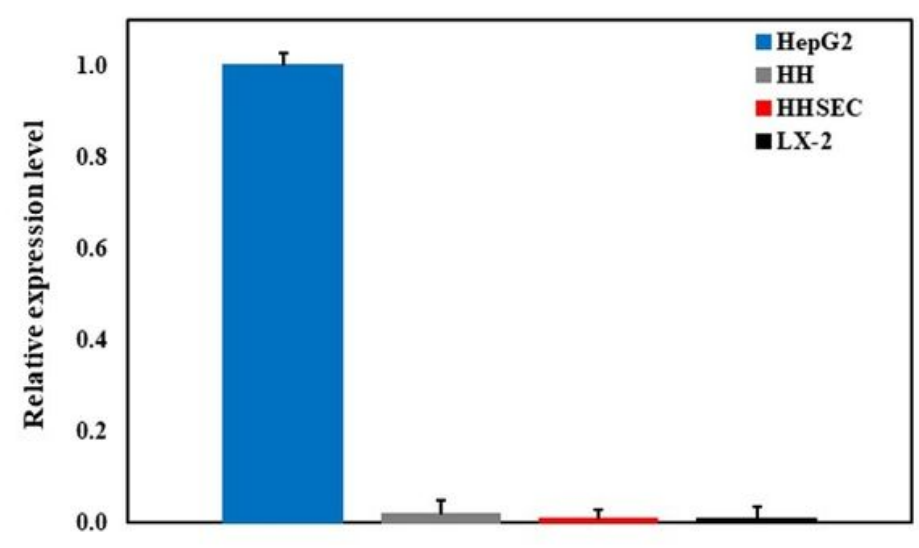

b

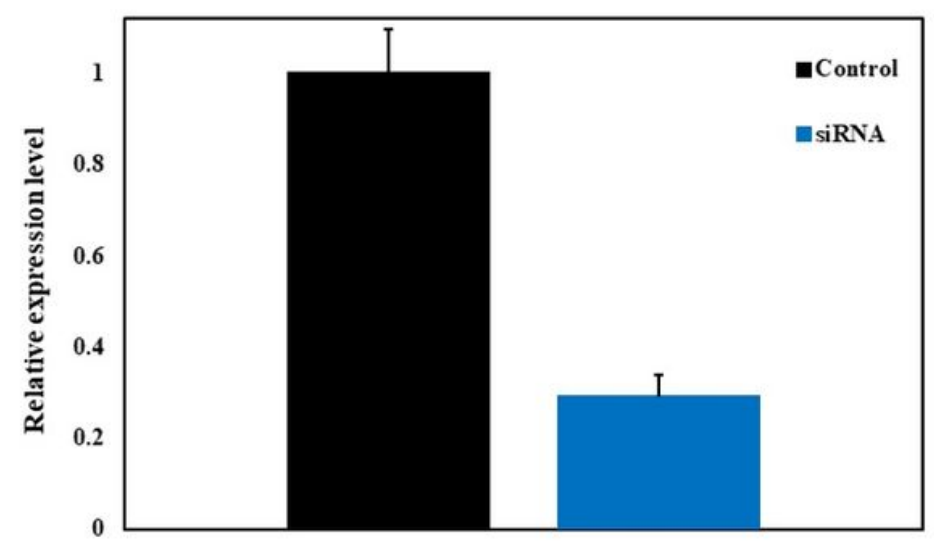

Figure 3

Relative expression levels of HSD11B2. a) The expression level of HSD11B2 in the HCC cancer cell line (HepG2) and three non-cancerous cell lines (HH, HHSEC, and LX-2) was measured using qPCR, and b) Confirmation of HSD11B2 knockdown using siRNA after $48 \mathrm{~h}$. Results are shown as mean \pm SEM.

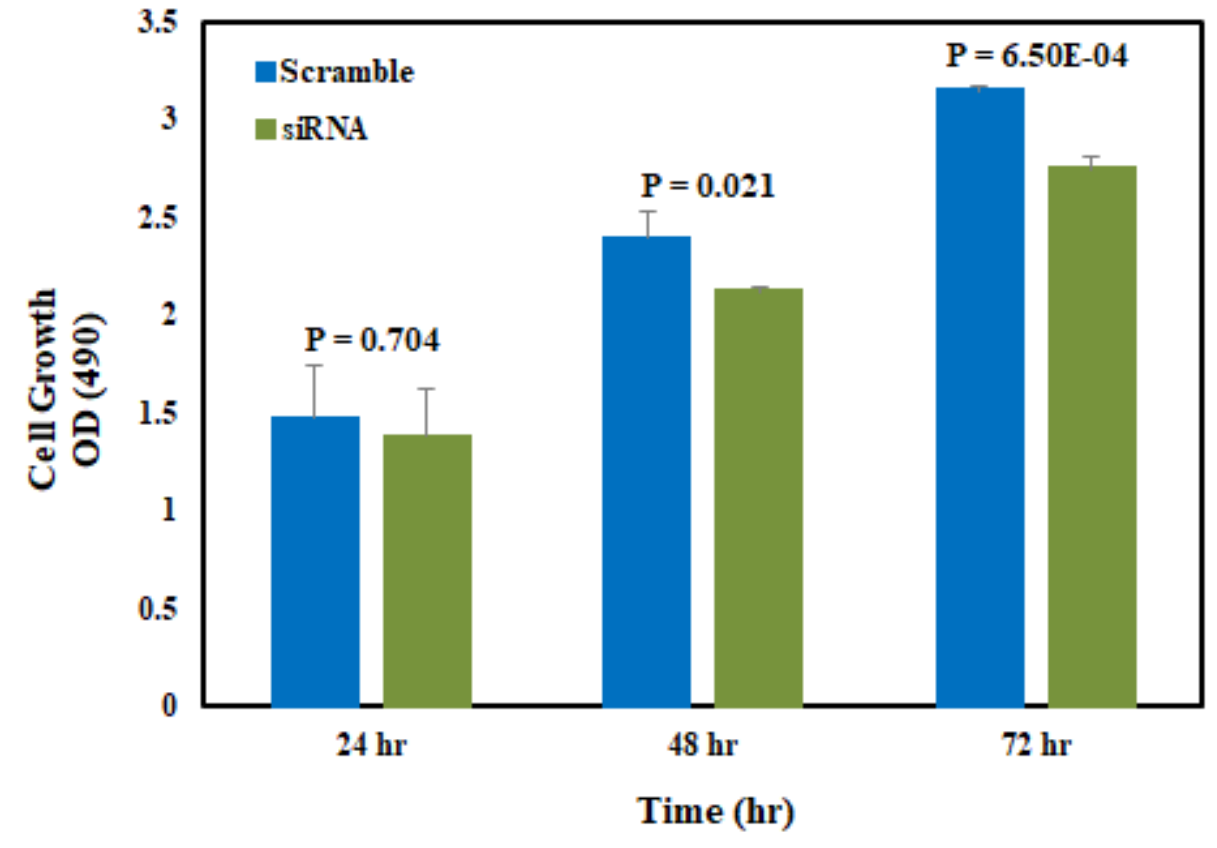

Figure 4

Effect of HSD11B2 knockdown on viability of HepG2 cells. MTS assay was used to assess cell viability (at $490 \mathrm{~nm}$ ). Results are shown as mean \pm SD. P-values were calculated using a two-sample t-test after log-transformation. 

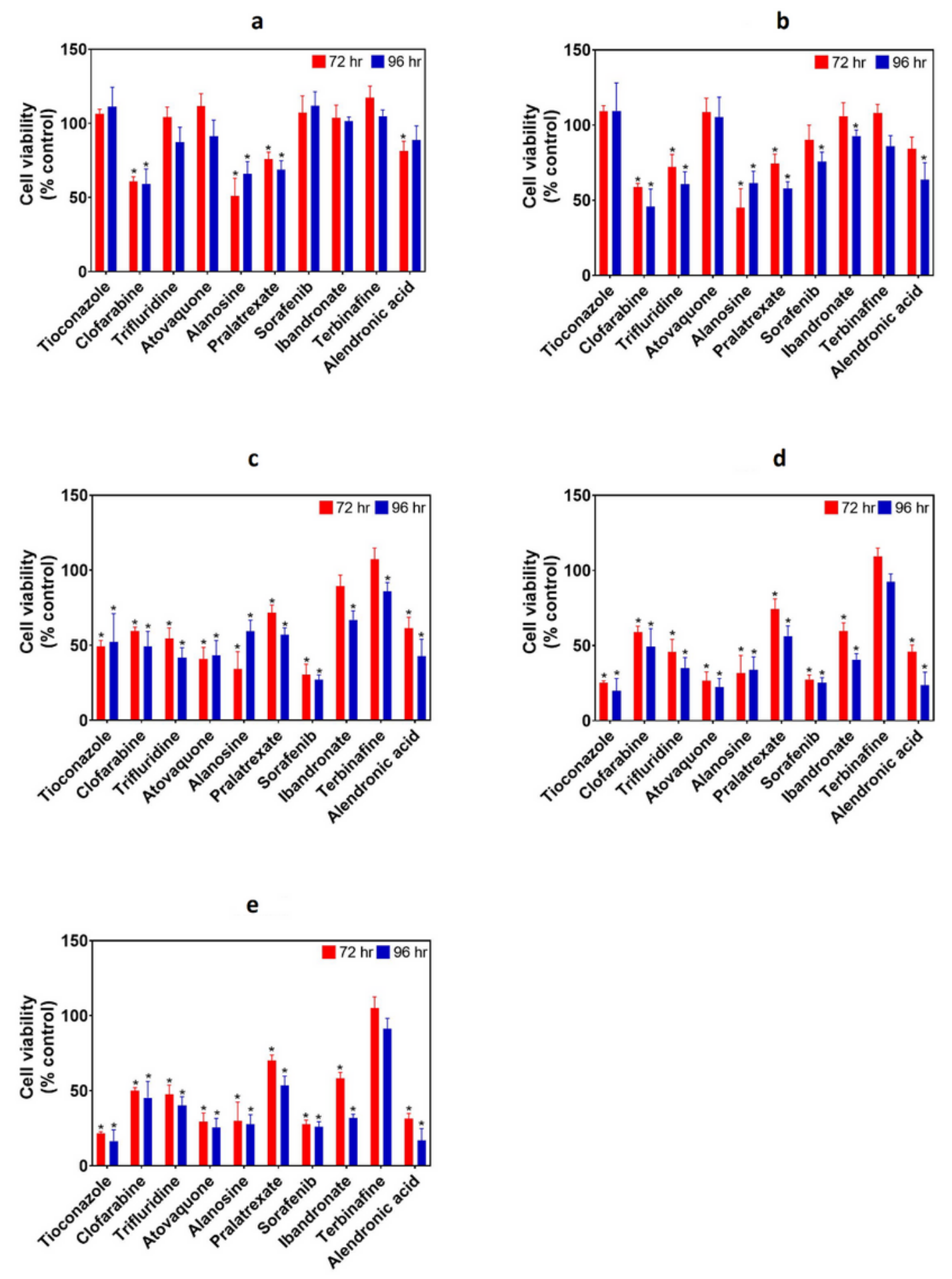

\section{Figure 5}

The effect of 9 metabolic drugs on the viability of HepG2 cells. Cell viability was measured by MTS assay at 5 concentrations (a) $100 \mathrm{nM},($ b) $1 \mu \mathrm{M}$, (c) $10 \mu \mathrm{M}$, (d) $25 \mu \mathrm{M}$ and (e) $50 \mu \mathrm{M}$, at 72 and 96 hours after treatment. All measurements were normalized to control absorption (0.5\% DMSO). The asterisks above each bar indicate the significance of viability compared to the control (calculated by two-sample t-test after log-transformation; $p<0.05)$. Results are shown as mean \pm SD. 


\section{Supplementary Files}

This is a list of supplementary files associated with this preprint. Click to download.

- SupplementaryfileS1.pdf 